\title{
The Magnetism-Nanostructure Interface in Advanced Magnetic Materials
}

\author{
David J. Sellmyer
}

Department of Physics and Astronomy and Center for Materials Research and Analysis, University of Nebraska, Lincoln, NE 68588-0113

The critical relationship between microstructure and nanostructure of materials and their magnetic properties has been appreciated for decades. Electromagnetic machinery, permanent magnets, and data recording and electronic devices all have seen steady and sometimes spectacular advances over this period. At the present time the most interesting research in magnetism and magnetic materials arises from new developments in structuring materials on the nanometer length scale. This talk will present recent advances and challenges in furthering this work, with particular attention paid to extremely high density magnetic recording films, exchange-coupled high-energy-product permanent-magnet materials, high-temperature permanent-magnet materials, and self-organized and patterned magnetic nanoarrays.

\section{$\underline{\text { Future High-Density Recording Media }}$}

Present longitudinal recording media are based mainly on CoCrPtX granular alloys and record at areal densities in the 20-50 gigabits per square inch $\left(\mathrm{Gb} / \mathrm{in}^{2}\right)$ region [1]. Such films are expected to be limited by superparamagnetic and noise considerations to densities of about $100-200 \mathrm{~Gb} / \mathrm{in}^{2}$. Further advances to 1 terabit/in ${ }^{2}\left(\mathrm{~Tb} / \mathrm{in}^{2}\right)$ are likely to be provided by high-anisotropy, small-grained films with perpendicular anisotropy [2]. Candidates include $\mathrm{L} 1_{0}$ ordered phases as FePt or CoPt, or $\mathrm{Co} / \mathrm{Pt}$ and related multilayers. Recent development of high-coercivity $(\sim 12 \mathrm{kOe})$, small-grain $(6-8$ $\mathrm{nm}$ ) films with minimal exchange coupling, and their study with HRTEM will be outlined [3].

\section{Exchange-Coupled Permanent Magnets}

The development of significantly stronger permanent magnets, with energy products in the range 75$100 \mathrm{MGOe}$, through discovery of new compounds or alloys is problematic. However, the concept of nanostructuring hard-soft nanocomposites on the exchange-length scale of about $10 \mathrm{~nm}$ is of great interest [4]. The difficulties in this approach involve inventing or adapting synthetic methods to achieve strong exchange coupling between the hard and soft phases, without grain growth of either phase beyond about $10 \mathrm{~nm}$. We have produced prototype $\mathrm{FePt}: \mathrm{Fe}_{1-\mathrm{x}} \mathrm{Pt}$ nanocomposites with energy products of about $53 \mathrm{MGOe}$, very close to those seen in the largest values known ( $\sim 54$ MGOe for $\mathrm{Nd}_{2} \mathrm{Fe}_{14} \mathrm{~B}$ ) [5]. New ideas and challenges in improving and studying nanostructures of this type will be discussed.

\section{$\underline{\text { High-Temperature Permanent Magnets }}$}

$\mathrm{Sm}_{2}(\mathrm{Co}, \mathrm{Fe}, \mathrm{Cu}, \mathrm{Zr})_{17}\left[\mathrm{Sm}_{2} \mathrm{TM}_{17}\right.$ or $\left.2: 17\right]$ magnets have been the best permanent magnets for use at temperatures up to about 300EC [6]. Their energy products of about $20 \mathrm{MGOe}$ at 300EC are significantly degraded at $\mathrm{T} \square$ 500EC where new applications are sought for the all-electric airplane. The cellular nanostructure of the 2:17 magnets consists of 2:17 grains surrounded by $\mathrm{Cu}$-containing $\mathrm{SmCo}_{5}$-structure boundaries. Recent research has shown a record high temperature coercivity (12.3 
$\mathrm{kOe}$ ) at 500EC in a Sm-Co-Ti-Cu alloy [7]. A physical model for this behavior and relationship to nanostructures as determined by TEM will be discussed.

\section{$\underline{\text { Self-Organized and Patterned Nanoarrays }}$}

A new topic of high interest is the study of magnetic nanoarrays by fabrication methods such as electrodeposition into self-assembled nanopore structures [8], laser-interference lithography [9], and focused-ion-beam synthesis [10]. Future applications have been suggested such as data storage, spin-logic devices, spin electronics, and quantum computing. If time permits one or more of these topics will be discussed.

This research is supported by DOE, NSF, ONR, ARO, AFOSR, NSIC and CMRA. It was performed in collaboration with R. Skomski, Y. Liu, M. Yan, Y. Qiang, and J. Zhou.

References

[1] See: Magnetic Storage Systems Beyond 2000, Ed. G.C. Hadjipanayis Series II, Vol. 41 (Kluwer, Dordrecht, 2001).

[2] M. H. Kryder, Ref. 1., p. 559.

[3] D.J. Sellmyer et al., IEEE Trans. Mag. 37 (2001) 1286.

[4] See: R. Skomksi and J.M.D. Coey, Permanent Magnetism (Inst. of Physics, Bristol, 1999), p. 298.

[5] D.J. Sellmyer, et al., in Handbook of Thin Films, Ed. H.S. Nalwa, Vol. 5: Nanomaterials and Magnetic Thin Films, (Academic Press, New York, 2002), p. 337.

[6] G.C. Hadjipanayis in Rare-Earth Iron Permanent Magnets, Ed., J.M.D. Coey (Oxford University Press, Oxford, 1996).

[7] J. Zhou et al., IEEE Trans. Mag. 37 (2001) 2518.

[8] D.J. Sellmyer et al., J. Phys. Cond. Matt. 13 (2001) R433.

[9] M. Zheng et al., Appl. Phys. Lett. 79 (2001) 2606.

[10] B.D. Terris et al., Appl. Phys. Lett. 75 (1999) 403. 